\title{
Human-animal chimeras for autologous organ transplantation: technological advances and future perspectives
}

\author{
Yingfei Lu ${ }^{1}$, Yu Zhou ${ }^{1,2}$, Rong Ju ${ }^{2}$, Jianquan Chen ${ }^{1,2}$ \\ ${ }^{1}$ Central Laboratory, Translational Medicine Research Center, ${ }^{2}$ Department of Obstetrics and Gynecology, The Affiliated Jiangning Hospital with \\ Nanjing Medical University, Nanjing 211100, China \\ Contributions: (I) Conception and design: All authors; (II) Administrative support: None; (III) Provision of study materials or patients: None; (IV) \\ Collection and assembly of data: None; (V) Data analysis and interpretation: None; (VI) Manuscript writing: All authors; (VII) Final approval of \\ manuscript: All authors. \\ Correspondence to: Jianquan Chen. Central Laboratory, Translational Medicine Research Center, The Affiliated Jiangning Hospital with Nanjing \\ Medical University, Nanjing 211100, China. Email: jqchen68@hotmail.com.
}

\begin{abstract}
Organ transplantation is the most promising curation for end-stage organ disease. However, the donor organ shortage has become a global problem that has limited the development of organ transplantation. Human-animal chimeras provide the ability to produce human organs in other species using autologous stem cells [e.g., induced pluripotent stem cells (iPSCs) or adult stem cells], which would be patient-specific and immune-matched for transplantation. Due to the potential application prospect of interspecies chimeras in basic and translational research, this technology has attracted much interest. This review focuses primarily on technological advances, including options of donor stem cell types and gene editing in donor cells and host animals, in addition to perspectives on human-animal chimeras in clinical and basic research.
\end{abstract}

Keywords: Autologous stem cells; embryo complementation; gene editing; human-animal chimeras; organ transplantation

Submitted Jul 17, 2019. Accepted for publication Sep 23, 2019.

doi: $10.21037 /$ atm.2019.10.13

View this article at: http://dx.doi.org/10.21037/atm.2019.10.13

\section{Introduction}

For patients suffering from end-stage organ failure, organ transplantation is their only hope for gaining a new life and has saved millions of lives worldwide. However, organ shortage and substantial risk of rejection have limited the development of organ transplantation. Even in the US, where over $50 \%$ of adults are registered as organ donors, more than 20 patients on the waiting list die every day. According to the speech delivered by Huang Jiefu, the Director of China National Organ Donation and Transplantation Committee, although China has become the world's second largest country for organ transplantation, the 20,201 transplant surgeries are mostly inadequate to meet the demand of over 300,000 organs per year. In 2015, voluntary donations became the only legitimate source for organ transplants in China. Meanwhile, the traditional culture makes recruitment of organ donors more challenging in China than other countries. Therefore, finding more resources for organ transplantation is urgently needed.

Emerging stem cell technology is promising for the development of tissue engineering and regenerative medicine as it can provide abundant biological materials. Using organs derived from the patients' cells [fibroblasts, adult stem cells, or induced pluripotent stem cells (iPSCs), etc.] as personalized medicine, is one of the trends in healthcare development. Organs generated with autologous cells minimize immune rejection, accordingly obviating the costs of the long-term administration of immunosuppression agents and reducing the risks of infection and tumorigenesis.

Scientists are trying to produce artificial organs by in vitro differentiation or 3-dimensional printing (1-3). Although stem cells can differentiate into many cell types 
in vitro, it is next to impossible to induce differentiation into multiple cell types simultaneously in the same dish. Moreover, the organ structures that consist of various cells and organ-specific molecules is too complex to simulate in vitro. Thus, the existing technology for artificial organs cannot meet the demand of transplantation, and is only suitable for disease modeling $(4,5)$ and drug screening (2). Before we can completely unveil the mysteries of reproduction and development, it is almost impossible to generate human organs in vitro for transplantation.

Fortunately, animal fetuses can provide a suitable environment for human organogenesis. Producing human cells, tissues, and whole organs in animals has been proven to be feasible. The animals bearing human organs or cells are known as human-animal chimeras. Using animals as biological incubators for human organs has become a new research trend because there are several benefits: (I) animals are plentiful and ready-to-use, and can allow both the patient and doctor to be well-prepared before transplantation; (II) the animal body provides a suitable niche for organogenesis, so the generated organs would be more natural and biological than the artificial organs produced in vitro; (III) the personally tailored organ derived from patient's stem cells may theoretically minimize the risk of immune rejection. Chimera research, was therefore, thought to be one of the hottest research areas according to Science Magazine in 2016. In this review, we summarize the experimental advances of interspecies chimeras, especially human-animal chimeras, including generation technique, options of donor stem cell types and gene editing in donor cells and host animals, in addition to perspectives on the potential roles in overcoming the limitations in organ transplantation by from organ shortage.

\section{Approaches to generation techniques}

Commonly used experimental approaches to acquire interspecies chimeras include aggregation of cleavage stage embryos, injection of pluripotent stem cells into blastocysts or morulae, and transplantation of adult stem cells into in utero fetus (Figure 1).

One way to introduce xenogeneic stem cells into an embryo is by blastocyst injection, which refers to injecting stem cells into in vivo/vitro-cultured blastocyst cavities. Ratmouse (6), human-mouse (7), and sheep-goat (8) chimeras with systemic chimerism are reported to be produced via blastocyst injection. However, blastocyst injection has failed to introduce stem cells into primate (such as rhesus monkey) embryos (9).

Chimeras may also result from the aggregation of two or more embryos either at equal or unequal stages. The quick and straightforward operation and low equipment cost make embryo aggregation an entry-level technique for chimera generation. Aggregation of rat-mouse (10), sheep-goat (11), and cattle-buffalo (12) embryos all form interspecies chimeras. Despite the lower survival rate of chimeric embryos produced by aggregation than by blastocyst injection, the chimerism rates have been observed to be higher $(13,14)$. Considering that chimerism rates decide whether the human-animal chimeras can be an organ resource, aggregation is also a desirable choice when the embryo and stem cells are in a good growth condition.

The in utero transplanting of stem cells was an in utero treatment for congenital diseases at first (15). Now, it has become the most widely used experimental technique in human-animal chimera study. Human adult stem cells, including mesenchymal stem cells (MSCs) and hematopoietic stem cells (HSCs), can form chimeras in mouse, rat, pig, sheep or goat fetuses and contribute to multiple organs of the chimeras (16-21). In utero transplantation is reported to be the most efficient way to limit human cells in the target organ of human-animal chimera (22).

However, the interspecies barrier between human and other animals is much thicker than that between rat and mouse. Even a minimal number of human cells may disrupt the development and growth of host animals. Consequently, the chimerism rates using the mentioned techniques are still extremely low. Contribution from human stem cells in human-mouse chimera generated by blastocyst injection was as low as $0.01 \%$ (23). Pigs, sheep, and goats are universally acknowledged as the preferred species for xenotransplantation into human body. However, due to the interspecies barriers, human cells only contributed $0.001 \%$ of the total cells in human-pig chimeric embryo (24) and up to $0.01 \%$ in human-sheep embryo according to the report in the 2018 AAAS annual meeting. This is far away from producing organs for transplantation into human bodies. Consequently, researchers have made much efforts to increase the chimerism rates by gene editing in host embryos and stem cells.

\section{Host embryo gene editing: embryo complementation}

One of the main ethical concerns raised by humananimal chimeras is how to avoid stem cell contribution 


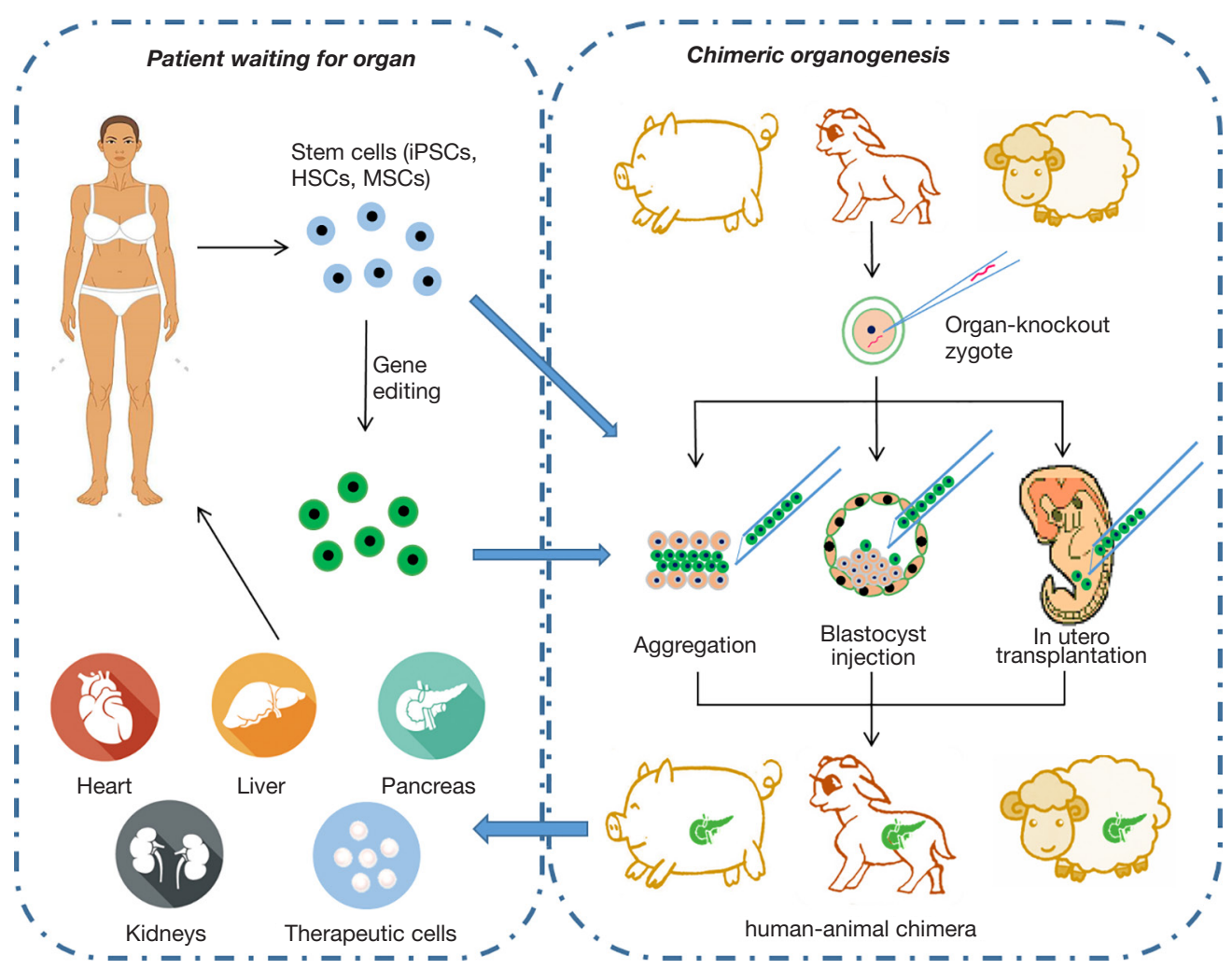

Figure 1 Interspecies chimeras may provide patient-specific organs for transplantation. The patient's stem cells (e.g., iPSCs, HSCs, MSCs) are injected into farm animal embryos to produce a human-animal chimera bearing needed organ for transplantation back into the patient's body. The chimeric animals can be made by inducing stem cells into the embryo through aggregation, blastocyst injection or in utero transplantation. Knocking out key regulators for organogenesis in zygote results in organ deficiency and provides vacancy for stem cells to produce a xenogenic organ. Meanwhile, gene editing in stem cells can trigger pathogenic gene switch-off, as well as the directed differentiation, contribution, and increased post-transplantation survival of the cells. iPSCs, induced pluripotent stem cells; HSCs, mesenchymal stem cells; MSCs, hematopoietic stem cells.

to the nervous or reproductive system. Modifying host embryos by knocking out the essential regulatory genes for target organs (e.g., Pdx1 for pancreas, Sal1 for kidneys, Rag2 for lymphocytes, Dcx for forebrain) in the zygotes leads to organ deficiency. This vacancy could be filled during development by introducing exogenous stem cells, producing chimeras in which the target organs mostly consist of injected stem cells (25-27). This technique, known as embryo complementation, was first used to search for the critical regulators of organ development (27). Through embryo complementation, researchers can limit the contribution of stem cells in a specific organ. This not only increases chimerism rates in target organs, but also minimizes the detrimental influence on host embryos from xenogenic cells and subsequently reduces the abortion and malformation rates.
If xenogenic stem cells are used for embryo complementation, a xenogenic organ would be developed and could form an interspecies chimera. In 2010, Nakauchi's group injected rat ESCs into Pdx1 knockout mice blastocysts. The rat ESCs were limited mostly to the pancreas and contributed to $81.9 \% \pm 3.4 \%$ of all cells in the pancreas (6). Chimeric mice remained alive and reached adulthood with no pancreas dysfunction. In 2017, this group successfully generated mouse pancreas in rats, the islets of which were transplanted into diabetic mice. No immune rejection was observed, and the blood glucose maintained at normal level (28).

However, it seems that blastocyst injection is not a suitable way for large human-animal chimeras. Although preliminary data confirmed that intraspecies porcine chimeras could be produced through blastocyst 
injection (29), injecting human PSCs resulted in systemic chimerism (unpublished data by Nakauchi's group). The cells at unexpected positions might cause malformation and provoke ethical concerns. On the contrary, in utero injection of PSCs to the site of organogenesis resulted in mainly local chimerism and less systemic chimerism (22).

To date, stem cell isolation and expansion in vitro is growing into maturity. Meanwhile, gene editing in sheep and porcine embryos are available using CRISPR-Cas9 (30,31). Moreover, eGenesis Bio has claimed that up to 62 genes could be batch edited, which would provide the feasibility of constructing host animals with deficiencies of all cell types in specific organs. These have all laid the roots for producing human organs in human-animal chimeras.

\section{Stem/progenitor cells for chimera production}

Theoretically, all types of cells with differentiation potency can contribute to the chimera. The chimerism rates depend on the potency of the cells. Embryonic stem cells (ESCs) and induced pluripotent stem cells (iPSCs) are the preferred cell types in chimera studies $(6,24,28,32)$. Due to their pluripotency, the incorporated stem cells contribute to multiple organs of the host animals. However, a recent study demonstrated that rhesus macaque ESCs were incapable of generating intraspecies chimeras by blastocyst injection (9). To explain this difference between rodent and primate stem cells, some researchers proposed that only naive ESCs are capable of producing chimeras with pre-implantation embryos (9), while primed ESCs (EpiSCs) are only chimera-competent in the epiblasts of post-transplantation embryos $(24,33)$. That is to say, the stem cells at a certain developmental stage are only competent in those embryos at an equal stage. Similarly, progenitors should be in utero transplanted into embryos at an equal stage (34), while adult stem cells should be injected to postnatal bodies to generate chimeras.

Nevertheless, several studies have demonstrated that human stem cells at asynchronous stages can also generate chimeras in animal embryos. Transplanting human adult stem cells (such as MSCs, neural stem cells and HSCs) into post-transplantation embryos of rat, sheep or goats $(17,19,35)$, and into murine blastocysts can all generate chimeras (36). Moreover, compared to iPSCs, autologous adult stem cells have advantages such as easy accessibility, simple culture conditions, and less tumorigenicity (37).

Several recent studies also demonstrated that despite the equality of the developmental stage, the survival of injected stem cells is also crucial for chimerism. Both rat and human EpiSCs overexpressing anti-apoptotic genes (e.g., BCL2, BCL-XL, CRMA, BMI1, etc.) can form interspecies chimeras in mouse embryos (38-40). Meanwhile, adding anti-apoptotic reagent Y27632 (ROCK1 inhibitor) into culture medium also gives rise to chimerism of primate EpiSCs into morulae (41). Therefore, other proliferationinducing and anti-apoptotic treatment, such as forced expression of SIRT1 (42), neurotrophin 3/4 (43) or Rho signaling pathway (44), might also impel chimera formation. Worryingly, however, apoptosis blockage in iPSCs might be more prone to tumorigenesis. In that case, more research is needed to assess the safety of increasing the chimerism rate through improving stem cell survival.

Gene editing in injected stem cells may trigger directed differentiation and guide contribution to specific tissues or organs. For example, Mixl1-overexpressed ESCs or iPSCs tended to contribute to endodermal organs after blastocyst injection (25). We speculate that overexpression of the critical regulators of organogenesis, such as $\mathrm{Pdx} 1$ and Sall 1 mentioned above, may also give rise to directed differentiation of stem cells. Moreover, gene editing has the capacity for pathogenic mutation correction in autologous stem cells. This would be beneficial for patients suffering from malignancy hematologic disease (45-47), and for those with end-stage organ failure caused by genetic disorders, such as polycystic renal disease and hepatolenticular degeneration, to receive autologous organs for transplantation.

\section{Facing challenges}

\section{Low rates of chimera and chimerism}

It is never easy to cross the xenobarrier. The more xenogenic cells contribute to the chimera, the higher risk of abortion and malformation (28). Only $20 \%$ of rat blastocysts injected with mouse ESCs became chimeric pups (6), while only $35 \%$ of all embryos transplanted in utero with human HSCs were eventually born alive $(18,35)$. By improving stem/progenitor cell survival, as we mentioned above, the rate of chimeric embryo occurrence increased.

Nonetheless, the impairment of host animals by xenogenic cells still exists and triggers adverse pregnancy outcomes. This might be due to undesired immunologic defense activation. In Ldlr, Rag2 and Il2rg KO mice, immunodeficiency allows injected human iPSCs and glial progenitor cells to contribute to mouse liver and brain, respectively $(48,49)$. However, the above techniques have 
only been proven to be effective in rodents. Methods to generate suitable human organs in livestock still lack sufficient evidence. Accordingly, further studies in large animals are urgently needed.

\section{Immune rejection}

The contamination of animal cells (e.g., endothelial cells) in the human organs produced by a chimera might lead to immune rejection. Although these cells could be eliminated by multiple gene knockouts in host embryos, the embryo development may also be severely affected. Knockout of $\alpha-1,3$-galactosyltransferase has been reported to be effective in minimizing immunogenicity of xenograft. The kidneys from $\alpha$-1,3-galactosyltransferase knockout pig worked properly with slight immunosuppressive therapy in a baboon body for up to 136 days (50). Overexpressing human CD46 exhibited a similar effect on avoiding immunogenicity for xenotransplantation (50-52). Excitingly, the latest research showed that xenotransplantation of a pig heart with $\alpha-1,3-$ galactosyltransferase-knockout combined with human CD46 and thrombomodulin overexpression functioned normally in baboons for up to 195 days (53). Can these genetically engineered pigs produce humanized organs more suitable for transplantation and prolong overall survival? This is a desirable orientation for chimera research.

\section{Virus infection risk}

Another fear is that human organs produced in animals might be transporters of new zoonoses, transferring endogenous retrovirus (ERV) to humans. Therefore, non-human primates, harboring pathogens that easily infect humans, are no longer thought to be promising organ donors for humans. In contrast, large farming animals, including pigs, sheep and goats are acknowledged as suitable xenogeneic donors for humans. Although there are still several kinds of ERVs in pigs which are infective for humans, scientists have been able to generate ERV-free pigs by using somatic cell nuclear transfer and CRISPR-Cas9 techniques (54). The ERV-free animals address this safety concern and offer novel options for animals bearing human organs.

\section{Economic benefits and future prospective}

Organ transplantation has proven to be the most economical treatment for organ failure. In Shanghai, kidney transplantation can save up to $¥ 150,000$ for each patient suffering from end-stage renal disease in the first 8 years (55). However, organ shortage and transplant rejection keep post-transplantation medical costs inflated. Interspecies chimeras provide the capability to produce adequate donor organ; thus, more patients on the waiting list can choose organ transplantation, and, more importantly, at earlier stage of disease. This would reduce the risk of severe complications.

Meanwhile, the autologous stem cell-derived organs may minimize the risk of rejection and consequently save the vast expense of immunosuppression and anti-infectives, which may cost up to $¥ 5,000-10,000$ per month. Moreover, the convenience of operating on animals would not only ensure full preoperative preparation of removing organs for chimeric animals and transplantation into the patients' bodies but also avoid organ necrosis during transportation. Also, producing patient-specific organs from autologous stem cell-animal chimera do not need in vitro organ culture, and thus may save significant expense compared to in vitro culture and differentiation of autologous iPSCs.

Apart from providing autologous organs, humananimal chimeras have additional benefits in clinical and experimental research. We might be able to produce therapeutic cells and functional proteins for clinical use. For instance, autologous HSCs in which pathogenic genes are edited might cure malignant hematological diseases with less immune rejection than allogeneic transplantation. Human cells overexpressing coagulation factor IX have been enriched in human-mouse chimeras, with the proteins being properly modified, secreted, and cleaved in vivo, thus being suitable for hemophilia B treatment (56). Although human-animal chimeras are still far away from clinical therapy, their application value in basic research has been undeniably proven in studies for human stem cell potency $(20,21)$, cell fate during embryo early development and organogenesis (21), as well as in vivo models of human diseases (35) for drug metabolism study $(57,58)$ and drug screening (48).

Crossing the species boundary between human and animals is always connected with many ethical issues. The possibility of inducing human cells into animal neural or reproductive systems has met particularly strong public resistance. However, human-animal chimera is not only a promising strategy to alleviate organ shortage, but also a reliable model for studies on organ development, pathogenesis, immunologic defense, and drug screening. Respecting the significance of human-animal chimera in basic and translational research, the National Institutes 
of Health (NIH) restarted funding for studies on adding human stem cells to animal embryos. Certainly, this research still needs to be cautiously carried out only after adequate studies in animal models and after undergoing more ethical review. US researchers are attempting to incubate human neurons for Parkinson's disease treatment in humanpig embryo, while a human-glial-progenitor-cell-mouse chimera was used as a research model for neurological and mental disorders several years ago (49,59). Furthermore, experiments have proven that human gametes crossing the interspecies reproductive barrier and hybridizing with gametes of animals, including anthropoid apes, is virtually impossible. In other words, even if human sperm or oocytes were to be produced in chimeras, no embryo with humanlike features would be capable of development.

Moreover, oocyte-like cells produced through in vitro differentiation have an impaired ability to achieve meiotic maturation, while the production of human gametes in animals would fill this gap. This would not only provide new tools for research on germ cell development and maturation but help patients with azoospermia or premature ovarian failure as well. In conclusion, rather than complete prohibition, which would obstruct the developmental possibilities of human-animal chimeras, continuing research under strict ethical review would be more beneficial to human society.

\section{Acknowledgments}

None.

\section{Footnote}

Conflicts of Interest: The authors have no conflicts of interest to declare.

Ethical Statement: The authors are accountable for all aspects of the work in ensuring that questions related to the accuracy or integrity of any part of the work are appropriately investigated and resolved.

\section{References}

1. Hindley CJ, Cordero-Espinoza L, Huch M. Organoids from adult liver and pancreas: Stem cell biology and biomedical utility. Dev Biol 2016;420:251-61.

2. Robertson MJ, Soibam B, O'Leary JG, et al. Recellularization of rat liver: An in vitro model for assessing human drug metabolism and liver biology. PLoS One 2018;13:e0191892.

3. Peng W, Datta P, Ayan B, et al. 3D bioprinting for drug discovery and development in pharmaceutics. Acta Biomater 2017;57:26-46.

4. Dutta D, Clevers H. Organoid culture systems to study host-pathogen interactions. Curr Opin Immunol 2017;48:15-22.

5. Dutta D, Heo I, Clevers H. Disease Modeling in Stem Cell-Derived 3D Organoid Systems. Trends Mol Med 2017;23:393-410.

6. Kobayashi T, Yamaguchi T, Hamanaka S, et al. Generation of rat pancreas in mouse by interspecific blastocyst injection of pluripotent stem cells. Cell 2010;142:787-99.

7. James D, Noggle SA, Swigut T, et al. Contribution of human embryonic stem cells to mouse blastocysts. Dev Biol 2006;295:90-102.

8. Polzin VJ, Anderson DL, Anderson GB, et al. Production of sheep $\leftrightarrow$ goat chimeras by blastocyst injection. Theriogenology 1986;25:183.

9. Tachibana M, Sparman M, Ramsey C, et al. Generation of chimeric rhesus monkeys. Cell 2012;148:285-95.

10. Bożyk K, Gilecka K, Humięcka M, et al. Mouse $\leftrightarrow$ rat aggregation chimaeras can develop to adulthood. Dev Biol 2017;427:106-20.

11. Jaszczak K, Parada R, Guszkiewicz A. Cytogenetic study of some tissues and age-related changes in cell proportions in a goat-sheep chimera. Cytogenet Cell Genet 1999;84:55-7.

12. Bian GH, Qin QL, Feng GX, et al. A Preliminary Study on Making Interspecific Chimeras between Cattle and Buffalo by Aggregating Blastomeres. China Animal Husbandry \& Veterinary Medicine 2007;34:44-6.

13. Guo J, Wu B, Li S, et al. Contribution of Mouse Embryonic Stem Cells and Induced Pluripotent Stem Cells to Chimeras through Injection and Coculture of Embryos. Stem Cells Int 2014;2014:409021.

14. Peli J, Schmoll F, Laurincik J, et al. Comparison of aggregation and injection techniques in producing chimeras with embryonic stem cells in mice. Theriogenology 1996;45:833-42.

15. Shaw SW, Bollini S, Nader KA, et al. Autologous transplantation of amniotic fluid-derived mesenchymal stem cells into sheep fetuses. Cell Transplant 2011;20:1015-31.

16. Chen X, Gong XL, Katsumata M, et al. Hematopoietic stem cell engraftment by early-stage in utero transplantation in a mouse model. Exp Mol Pathol 
2009;87:173-7.

17. Chen CP, Liu SH, Huang JP, et al. Engraftment potential of human placenta-derived mesenchymal stem cells after in utero transplantation in rats. Hum Reprod 2009;24:154-65.

18. Zeng F, Chen MJ, Baldwin DA, et al. Multiorgan engraftment and differentiation of human cord blood CD34+ Lin- cells in goats assessed by gene expression profiling. Proc Natl Acad Sci U S A 2006;103:7801-6.

19. Liechty KW, MacKenzie TC, Shaaban AF, et al. Human mesenchymal stem cells engraft and demonstrate sitespecific differentiation after in utero transplantation in sheep. Nat Med 2000;6:1282-6.

20. Fujiki Y, Fukawa K, Kameyama K, et al. Successful multilineage engraftment of human cord blood cells in pigs after in utero transplantation. Transplantation 2003;75:916-22.

21. Sun Y, Xiao D, Pan XH, et al. Generation of human/ rat xenograft animal model for the study of human donor stem cell behaviors in vivo. World J Gastroenterol 2007;13:2707-16.

22. Suchy F, Nakauchi H. Interspecies chimeras. Curr Opin Genet Dev 2018;52:36-41.

23. Theunissen TW, Friedli M, He Y, et al. Molecular Criteria for Defining the Naive Human Pluripotent State. Cell Stem Cell 2016;19:502-15.

24. Wu J, Platero-Luengo A, Sakurai M, et al. Interspecies Chimerism with Mammalian Pluripotent Stem Cells. Cell 2017;168:473-86.e15.

25. Kobayashi T, Kato-Itoh M, Nakauchi H. Targeted organ generation using Mixl1-inducible mouse pluripotent stem cells in blastocyst complementation. Stem Cells Dev 2015;24:182-9.

26. Usui J, Kobayashi T, Yamaguchi T, et al. Generation of kidney from pluripotent stem cells via blastocyst complementation. Am J Pathol 2012;180:2417-26.

27. Chen J, Lansford R, Stewart V, et al. RAG-2-deficient blastocyst complementation: an assay of gene function in lymphocyte development. Proc Natl Acad Sci U S A 1993;90:4528-32.

28. Yamaguchi T, Sato H, Kato-Itoh M, et al. Interspecies organogenesis generates autologous functional islets. Nature 2017;542:191-6.

29. Matsunari H, Nagashima H, Watanabe M, et al. Blastocyst complementation generates exogenic pancreas in vivo in apancreatic cloned pigs. Proc Natl Acad Sci U S A 2013;110:4557-62.

30. Wu J, Vilarino M, Suzuki K, et al. CRISPR-Cas9 mediated one-step disabling of pancreatogenesis in pigs. Sci Rep 2017;7:10487.

31. Vilarino M, Rashid ST, Suchy FP, et al. CRISPR/Cas9 microinjection in oocytes disables pancreas development in sheep. Sci Rep 2017;7:17472.

32. Simerly C, McFarland D, Castro C, et al. Interspecies chimera between primate embryonic stem cells and mouse embryos: monkey ESCs engraft into mouse embryos, but not post-implantation fetuses. Stem Cell Res 2011;7:28-40.

33. Wu J, Greely HT, Jaenisch R, et al. Stem cells and interspecies chimaeras. Nature 2016;540:51-9.

34. Cohen MA, Wert KJ, Goldmann J, et al. Human neural crest cells contribute to coat pigmentation in interspecies chimeras after in utero injection into mouse embryos. Proc Natl Acad Sci U S A 2016;113:1570-5.

35. Zeng F, Huang SZ, Gong ZJ, et al. Long-term deregulated human hematopoiesis in goats transplanted in utero with BCR-ABL-transduced lin(-)CD34(+) cord blood cells. Cell Res 2013;23:859-62.

36. Harder F, Henschler R, Junghahn I, et al. Human hematopoiesis in murine embryos after injecting human cord blood-derived hematopoietic stem cells into murine blastocysts. Blood 2002;99:719-21.

37. Jiang Y, Jahagirdar BN, Reinhardt RL, et al. Pluripotency of mesenchymal stem cells derived from adult marrow. Nature 2002;418:41-9.

38. Wang X, Li T, Cui T, et al. Human embryonic stem cells contribute to embryonic and extraembryonic lineages in mouse embryos upon inhibition of apoptosis. Cell Res 2018;28:126-9.

39. Masaki H, Kato-Itoh M, Takahashi Y, et al. Inhibition of Apoptosis Overcomes Stage-Related Compatibility Barriers to Chimera Formation in Mouse Embryos. Cell Stem Cell 2016;19:587-92.

40. Huang K, Zhu Y, Ma Y, et al. BMI1 enables interspecies chimerism with human pluripotent stem cells. Nat Commun 2018;9:4649.

41. Kang Y, Ai Z, Duan K, et al. Improving Cell Survival in Injected Embryos Allows Primed Pluripotent Stem Cells to Generate Chimeric Cynomolgus Monkeys. Cell Reports 2018;25:2563-76.e9.

42. Jang J, Huh YJ, Cho HJ, et al. SIRT1 Enhances the Survival of Human Embryonic Stem Cells by Promoting DNA Repair. Stem Cell Reports 2017;9:629-41.

43. Pyle AD, Lock LF, Donovan PJ. Neurotrophins mediate human embryonic stem cell survival. Nat Biotechnol 2006;24:344-50.

44. Ohgushi M, Minaguchi M, Sasai Y. Rho-Signaling- 
Directed YAP/TAZ Activity Underlies the Long-Term Survival and Expansion of Human Embryonic Stem Cells. Cell Stem Cell 2015;17:448-61.

45. De Ravin SS, Li L, Wu X, et al. CRISPR-Cas9 gene repair of hematopoietic stem cells from patients with $\mathrm{X}$-linked chronic granulomatous disease. Sci Transl Med 2017. doi: 10.1126/scitranslmed.aah3480.

46. Ye L, Wang J, Tan Y, et al. Genome editing using CRISPR-Cas9 to create the HPFH genotype in HSPCs: An approach for treating sickle cell disease and betathalassemia. Proc Natl Acad Sci U S A 2016;113:10661-5.

47. Hoban MD, Lumaquin D, Kuo CY, et al. CRISPR/Cas9Mediated Correction of the Sickle Mutation in Human CD34+ cells. Mol Ther 2016;24:1561-9.

48. Yang J, Wang Y, Zhou T, et al. Generation of Human Liver Chimeric Mice with Hepatocytes from Familial Hypercholesterolemia Induced Pluripotent Stem Cells. Stem Cell Reports 2017;8:605-18.

49. Han X, Chen M, Wang F, et al. Forebrain engraftment by human glial progenitor cells enhances synaptic plasticity and learning in adult mice. Cell Stem Cell 2013;12:342-53.

50. Iwase H, Liu H, Wijkstrom $M$, et al. Pig kidney graft survival in a baboon for 136 days: longest life-supporting organ graft survival to date. Xenotransplantation 2015;22:302-9.

51. Mohiuddin MM, Corcoran PC, Singh AK, et al. B-cell depletion extends the survival of GTKO.hCD46Tg pig heart xenografts in baboons for up to 8 months. Am J Transplant 2012;12:763-71.

Cite this article as: Lu Y, Zhou Y, Ju R, Chen J. Humananimal chimeras for autologous organ transplantation: technological advances and future perspectives. Ann Transl Med 2019;7(20):576. doi: 10.21037/atm.2019.10.13
52. van der Windt DJ, Bottino R, Casu A, et al. Long-term controlled normoglycemia in diabetic non-human primates after transplantation with hCD46 transgenic porcine islets. Am J Transplant 2009;9:2716-26.

53. Längin M, Mayr T, Reichart B, et al. Consistent success in life-supporting porcine cardiac xenotransplantation. Nature 2018;564:430-3.

54. Niu D, Wei HJ, Lin L, et al. Inactivation of porcine endogenous retrovirus in pigs using CRISPR-Cas9. Science 2017;357:1303-7.

55. Wang W, Liang H, Lu W. Analysis on treatment burden of end-stage renal disease patients and related policy suggestions. Chin Heal Res 2018:121-6.

56. Yang CM, Gong XL, Qiu J, et al. Engraftment of genetically modified human amniotic fluid-derived progenitor cells to produce coagulation factor IX after in utero transplantation in mice. Cell Biol Int 2013;37:420-9.

57. Pozo OJ, Lootens L, Van Eenoo P, et al. Combination of liquid-chromatography tandem mass spectrometry in different scan modes with human and chimeric mouse urine for the study of steroid metabolism. Drug Test Anal 2009; 1:554-67.

58. Botrè F. Humanized animal models to study drug metabolism: no longer a "chimera"? Clin Chem 2009;55:1763-4.

59. Windrem MS, Osipovitch M, Liu Z, et al. Human iPSC Glial Mouse Chimeras Reveal Glial Contributions to Schizophrenia. Cell Stem Cell 2017;21:195-208.e6. 